\title{
Formal Modeling of Cyber-Physical Systems: Lessons Learn from Refinement and Proof Based Methods
}

\author{
Yamine Ait Ameur ${ }^{(凶)}$ \\ Institut National Polytechnique, Toulouse, France \\ yamine@n7.fr
}

\begin{abstract}
Cyber-Physical Systems refer to the tight integration and coordination between computational and physical resources. Modeling their behavior requires handling continuous and discrete behaviors. The definition of the associated models refers to continuous and discrete systems theories. In this talk, we address the problem of designing correct software to control cyber-physical systems. We recall the necessary basic concepts allowing a designer to model such systems. We also give an overview of the different formal approaches supporting the formal verification of these hybrid systems and we highlight the results obtained using these techniques. In particular, we focus on the use of proof and refinement based methods.
\end{abstract}

